\title{
Is cooking food in iron pots an appropriate solution for the control of anaemia in developing countries? A randomised clinical trial in Benin
}

\author{
Waseem Sharieff $1,2,3,4$, Joyce Dofonsou ${ }^{5}$ and Stanley Zlotkin ${ }^{6, *}$ \\ 'Radiation Oncology Programme, McMaster University, Hamilton, Ontario, Canada: ${ }^{2}$ Department of Health \\ Policy, Management and Evaluation, University of Toronto, Toronto, Ontario, Canada: ${ }^{3}$ The Hospital for Sick \\ Children, Research Institute, University of Toronto, Toronto Ontario, Canada: ${ }^{4}$ Medical Advisory Secretariat, \\ Ministry of Health and Long Term Care, Government of Ontario, Canada: ${ }^{5}$ CARE Benin, Porto Novo, Benin: \\ ${ }^{6}$ Departments of Paediatrics, Public Health Sciences, and Nutritional Sciences, The Hospital for Sick Children, \\ Research Institute, University of Toronto, 555 University Avenue \# 8260, Toronto, Ontario, Canada, M5G 1X8
}

Submitted 20 March 2007: Accepted 23 July 2007: First published online 15 0ctober 2007

\begin{abstract}
Objective: To evaluate the effect of two types of iron pots on haemoglobin ( $\mathrm{Hb})$ and serum ferritin (SF) concentrations in young children (6-24 months), adolescent girls (11-15 years) and women of reproductive age (15-44 years), whose households were provided with iron pots for cooking food over a period of 6 months, compared with controls.

Design and methods: We randomly assigned 161 households including 339 individuals from the three subgroups to cast iron pots, blue steel pots or oral iron supplements (control). In the control group, children received micronutrient Sprinkles $^{\mathrm{TM}}$, and adolescent girls and women received iron tablets daily for 6 months. We measured $\mathrm{Hb}, \mathrm{SF}$ and C-reactive protein concentrations at baseline and 6 months, and compared groups using regression models.

Results: Anaemia prevalence $\left(\mathrm{Hb}<110 \mathrm{gl}^{-1}\right.$ in infants, $\mathrm{Hb}<120 \mathrm{gl}^{-1}$ in girls or women) was $47 \%$ (cast iron group), 50\% (blue steel group) and 50\% (control) at baseline. At 6 months, there were no significant differences in $\mathrm{Hb}$ concentrations among groups; however, differences in SF concentrations were significant $(P<0.0001)$ - the control group had higher SF concentrations compared with the groups using iron pots. Also, differences in the prevalence of iron-deficiency anaemia (IDA; anaemia plus $\mathrm{SF}<15 \mu \mathrm{gl} \mathrm{l}^{-1}$ ) were almost significant between cast iron and control groups $(P=0.08)$, and blue steel and control groups $(P=0.05)$. Conclusion: There is no evidence that iron cooking pots are effective against IDA. Further research is warranted to determine whether the iron leached from the pots is bioavailable.
\end{abstract}

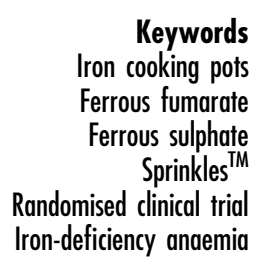

There have been a few studies which have examined the impact of the use of iron cooking pots on anaemia status in at-risk populations. For example, Adish et al. randomly assigned 407 young Ethiopian children (2-5 years) to iron cooking pots or aluminium pots, and demonstrated that iron pots were efficacious in reducing the prevalence of anaemia ${ }^{1}$. They suggested that all the members of the household are likely to benefit from eating food cooked in a single pot over a long period of time, and that cooking in iron pots could be a cost-effective strategy to control iron-deficiency anaemia (IDA). Borigato and Martinez found similar results in a study of 45 preterm, 4-month-old Brazilian children $^{2}$.
Intrigued by these encouraging results, the Canadian International Development Agency (CIDA) requested proposals to evaluate iron cooking pots, in relation to other interventions, for the control of anaemia and IDA in young children, adolescent girls and women of reproductive age in developing countries. CIDA allocated a total funding of CAN\$1.9 million and contracted a consortium comprising the University of Toronto, CARE Canada and PATH Canada to carry out these evaluations in Benin and Vietnam. The project was named 'Appropriate Solutions for Anemia Control (ASAC)' and was to be completed in two phases: phase 1 was intended to evaluate the efficacy, feasibility, acceptability and cost-effectiveness of iron pots compared with other 
interventions; phase 2 was meant to reach up to 100000 beneficiaries with the most cost-effective strategy identified in phase 1 and further evaluate the effectiveness and cost-effectiveness of that strategy.

In the present paper we report the results of the efficacy study which was carried out in Benin. The primary objective of this study was to evaluate the effect of two different forms of iron pots on haemoglobin $(\mathrm{Hb})$ and serum ferritin (SF) concentrations in young children, adolescent girls and women of reproductive age, whose households were provided with iron pots for cooking food over a period of 6 months, compared with controls. Secondary objectives were: (1) to evaluate the effect of iron pots on anaemia and IDA in relation to controls; and (2) to determine compliance in each group.

\section{Methods}

\section{Study area, population and recruitment}

The study took place in the town of Porto Novo, on the Atlantic coast of Benin, between October 2004 and March 2005. Benin is a low-income country in Africa - the population density is 57 per $\mathrm{km}^{2}$ and $42 \%$ of the population lives in the cities. Anaemia prevalence is 42.0 to $73.6 \% \%^{3,4}$; it is higher in children and women of reproductive age and $^{3}$ an of anaemia cases are due to iron deficiency ${ }^{4}$. Families live in housing compounds and share food and other aspects of daily living. We contacted heads of the families to introduce the study and obtain consent for participation if they were eligible. One of the eligibility criteria was that households must include individuals from at least two of the following subgroups: women of reproductive age (15-44 years old), infants (6-24 months old) and adolescent girls (11-15 years old); i.e. one woman plus one infant, or one woman plus one adolescent girl. Other criteria were that individuals must not be taking iron supplementation or have received a blood transfusion, must be willing to stay in the study area for at least 6 months, must provide informed consent, and must have $\mathrm{Hb}$ concentration $>70 \mathrm{gl}^{-1}$.

The study was a cluster-randomised clinical trial. Housing compounds (clusters) containing two participants from the three subgroups were randomly assigned to one of the three intervention groups using a random digit generator. Out of the three interventions, two used an iron cooking pot made of either cast iron or blue steel. The third group (control) received Sprinkles ${ }^{\mathrm{TM}}$ for young children and iron tablets for adolescent girls and women of reproductive age. Sprinkles ${ }^{\mathrm{TM}}$ were provided as single-dose sachets which included $12.5 \mathrm{mg}$ of iron as microencapsulated ferrous fumarate (Descote Ferrous Fumarate 60; Particle Dynamics Inc.) and $5 \mathrm{mg}$ of zinc as zinc gluconate. Each caregiver was instructed to add the contents of a Sprinkles ${ }^{\mathrm{TM}}$ sachet to the child's meal serving (after the meal was cooked) once daily for the 6-month period at any mealtime. Girls and women were instructed to take two tablets ( $60 \mathrm{mg}$ of iron) daily for 3 months followed by one tablet ( $30 \mathrm{mg}$ of iron) daily for another 3 months. Iron tablets contained iron in the form of ferrous sulfate and were supplied by the Laboratoire Internationale O.H. Inc. Iron pots were procured in Vietnam and shipped to Benin. Each had a capacity of 2 litres and weighed 7 lbs.

The primary outcomes were $\mathrm{Hb}$ and SF concentrations at 6 months from baseline. Secondary outcomes included anaemia and IDA at 6 months; and compliance during the 6-month study period. Anaemia was defined as $\mathrm{Hb}<110 \mathrm{gl}^{-1}$ in children, and $\mathrm{Hb}<120 \mathrm{gl}^{-1}$ in girls or women. IDA was defined as anaemia plus $\mathrm{SF}<15 \mathrm{\mu gl}^{-1}$.

We randomised by housing compounds to minimise cross-contamination due to food sharing within housing compounds. Those performing the statistical analyses were unaware of group assignments until the code was broken upon the completion of data analyses.

\section{Data collection}

At baseline, fieldworkers visited homes and used structured pre-coded forms to collect information. Trained technicians collected venous blood, a drop of which was used to measure $\mathrm{Hb}$ concentration with a portable Hemocue B-Hb photometer ${ }^{5}$. The rest of the sample was sent to a local laboratory for the analyses of SF and C-reactive protein (CRP) concentrations using Immulite 1 (Diagnostic Products Corp.).

From baseline to 6 months, fieldworkers visited participants at their homes once every two weeks for a total of 12 follow-up visits. At each visit, fieldworkers completed structured pre-coded forms regarding compliance and adverse effects over the preceding two weeks, counted the number of unused sachets/tablets and recorded the count on the forms, and provided caregivers with verbal educational support and advice regarding any concerns. Fieldworkers also asked the caregivers whether they shared any of the assigned sachets or tablets with other family members and instructed them to give all sachets or tablets to the participating individual.

At 6 months, trained technicians repeated all biochemical measurements.

\section{Data processing}

Data were entered into Epi Info ${ }^{\mathrm{TM}}$ version 3.3 (Centers for Disease Control and Prevention) and sent electronically to the study centre at The Hospital for Sick Children in Toronto, Ontario, Canada where data were checked for numeric and logical consistency before analyses. Any discrepancy was resolved by sending queries to the study site. To account for the presence of infection ${ }^{6}$, we dichotomised CRP concentrations using a cut-off level of $>8.3 \mathrm{mgl}^{-1}$. To estimate compliance, we calculated the 
number of weekly uses of pots per household in the iron pot groups; in the control group, we calculated the percentage of empty sachets (or used tablets) out of the total assigned for each participant.

\section{Power calculations}

Power calculations were based on comparing $\mathrm{Hb}$ concentrations among groups. We considered a $5 \mathrm{gl}^{-1}$ difference in $\mathrm{Hb}$ means between any two groups at the end of the study as clinically meaningful. Thus, 92 to 103 participants per group had power in the range of $80-85 \%$ to reject the hypothesis that the difference in $\mathrm{Hb}$ means between any of the pot groups and the control group was zero $(\alpha=0.05)$.

\section{Statistical analyses}

Preliminary analyses involved examining the data with descriptive statistics and histograms. The distribution of SF concentrations was skewed, which we normalised by taking the natural logarithm. To compare groups for outcomes (end values of $\mathrm{Hb}, \mathrm{SF}$, anaemia and IDA), we used random effects linear regression models for continuous variables and random effects logistic regression models for binary variables. These models had end values (outcome) as the dependent variable; group allocation and baseline values (of the outcome variable) as independent variables; and housing compound as random effects. Thus, the model assumed a correlation between participants within a housing compound. We also examined the effect of potential confounders, such as infection, by adding dichotomised CRP as a covariate to these models (since SF is sensitive to infection) ${ }^{7}$.

In addition, we carried out subgroup analyses which were an extension of the above models and included an age subgroup (infant, girls and women) as a covariate with an interaction term between the group allocation and subgroup. Thus, these models simultaneously tested whether: (1) the effect on an outcome depended upon group allocation; (2) the effect depended on baseline values of that outcome variable; and (3) the effect for a group was different across subgroups.

To examine compliance, we used cumulative histograms. Our analyses were based on the intention-to-treat principle. We considered $P<0.05$ as significant for hypothesis testing and used Tukey's adjustment for multiple comparisons. We used SAS version 9.1 (SAS Institute) for data processing, power calculations, and statistical and graphical analyses.

\section{Ethics approval}

The research ethics committees at The Hospital for Sick Children, Toronto, Canada and the Ministry of Public Health, Benin approved the study. Prior to the intervention, fieldworkers obtained informed and signed consent from mothers of the participating children and individually from adolescent girls and women.

\section{Results}

\section{Recruitment}

A total of 426 participants from 213 housing compounds were assessed for eligibility, of whom 339 participants (161 housing compounds) entered the study. Because of difficulties in collecting or processing blood samples, 45 participants had missing data for biochemical indices. Thus, we had complete biochemical data for 294 (86\%) participants. The trial profile is shown in Fig. 1.

\section{Baseline characteristics}

Baseline characteristics of the participants on whom complete data were available are shown in Table 1. As can be seen, the baseline characteristics among the three groups were similar.

\section{Outcomes}

At the end of the study, differences in mean $\mathrm{Hb}$ concentrations or anaemia prevalence between any two groups were not significant (Table 2). However, SF concentrations were significantly different across groups $(P<0.0001)$; the model adjusted for baseline SF and CRP concentrations. Also, differences in IDA prevalence between cast iron and control groups $(P=0.08)$, and between blue steel and control groups $(P=0.05)$, were close to significant levels.

Subgroup analyses revealed that the effect on any of the outcomes in a group did not differ significantly by age subgroups.

\section{Compliance and adverse events}

Figure 2 shows compliance in the three groups. In the iron pot groups, pots were used in $60 \%$ of the households at least three times a week; $50 \%$ of children in the control group consumed at least 60 sachets, $50 \%$ of adolescent girls and 50\% of women consumed at least 60 tablets.

No adverse events were reported during the study.

\section{Discussion}

We studied the effect of iron cooking pots as a household intervention on haematological indices in young children, adolescent girls and women of reproductive age in relation to interventions of proven efficacy - micronutrient Sprinkles $^{\mathrm{TM}}$ for young children and ferrous sulphate tablets for adolescent girls and women. In a population with a moderate prevalence of anaemia ( 50\%), the use of iron pots (blue steel or cast iron) was not effective in 


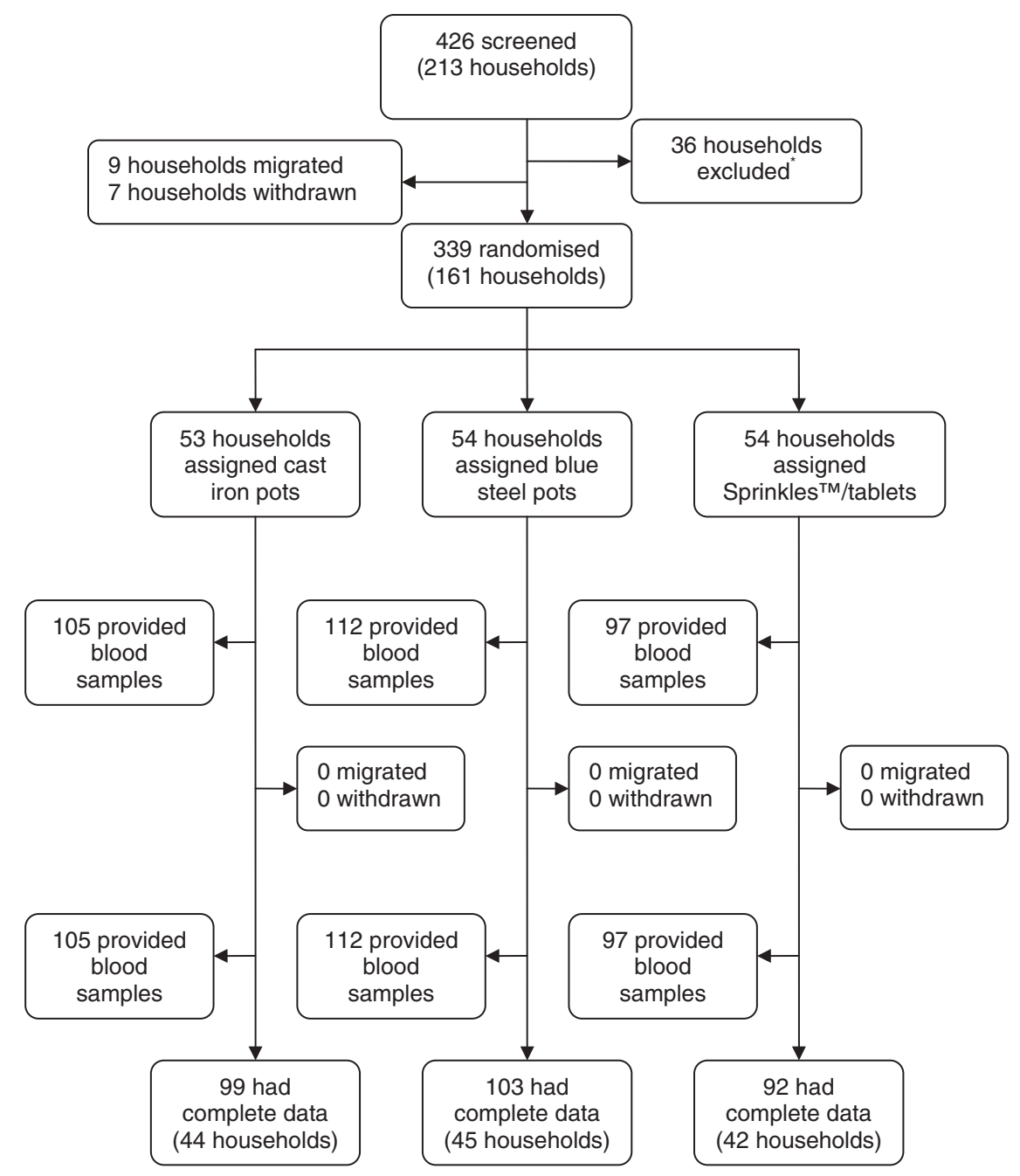

Fig. 1 Trial profile. *Thirty-six households excluded for not having a woman of reproductive age plus an infant or adolescent girl, families planning to move out of the study area, any individual with $\mathrm{Hb}<70 \mathrm{gl}^{-1}$ or not consenting

controlling anaemia. The effect on haematological indices was inferior in the iron pot groups relative to the control group (as evidenced by significant differences in SF concentrations among groups).

Our results cannot be directly compared with previous studies of iron pots which did not include adolescent girls and women of reproductive age. Adish et al. and Borigato and Martinez reported a significant change in $\mathrm{Hb}$ concentrations and anaemia prevalence in young children in the iron pot group ${ }^{1,2}$. The first study reported that anaemia prevalence dropped from $57 \%$ at baseline to $13 \%$ at the end of 12 months. However, the authors did not stratify the cohort by iron status and presented a brief description of their ferritin values; there was no indication of whether they used a marker to account for falsely elevated SF concentrations and thus it is difficult to infer that the reduction in anaemia prevalence in their study was due to improvement in iron status by using iron pots. The second study stratified by iron status but had a small sample size (about 23 per group). Furthermore, the authors studied preterm infants, all of whom received iron supplements (in addition to food cooked in iron pots).

Similarly, our results cannot be directly compared with those of Geerligs et al., who reported improvement in iron status - by virtue of significant differences in zinc protoporphyrin values - between iron pot and aluminium pot groups at the end of 20 weeks in a subgroup of children $<12$ months of age who were consistent users of the pots ${ }^{8}$. This finding may be due to the small sample size ( 9 [iron pot] vs. 14 [aluminium pot]) in this subgroup, which enhanced the chance of obtaining a significant result by inflating the type one error rate in their subgroup analyses; however, in both groups zinc protoporphyrin increased significantly from baseline - this could be due to worsening of iron deficiency (the authors used a cut-off level of $>3.1 \mu \mathrm{gg}^{-1} \mathrm{Hb}$ to define iron deficiency), or could be due to a confounder such as 
Table 1 Participants' characteristics

\begin{tabular}{|c|c|c|c|}
\hline & Cast iron & Blue steel & Control \\
\hline No. of households & 44 & 45 & 42 \\
\hline \multicolumn{4}{|c|}{ No. of people/household* } \\
\hline$<5$ & $10(22)$ & $9(20)$ & $9(21)$ \\
\hline $5-10$ & $33(75)$ & $32(71)$ & $27(65)$ \\
\hline$<10$ & $1(3)$ & $4(9)$ & $6(14)$ \\
\hline \multicolumn{4}{|l|}{ Age (years)† } \\
\hline Infants & $1 \pm 0.5[26]$ & $1 \pm 0.3[24]$ & $1 \pm 0.4[21]$ \\
\hline Girls & $13 \pm 1[29]$ & $13 \pm 1[34]$ & $13 \pm 1[29]$ \\
\hline Women & $31 \pm 8[44]$ & $32 \pm 10[45]$ & $32 \pm 8[42]$ \\
\hline Women interviewed* & $44(100)$ & $45(100)$ & $42(100)$ \\
\hline \multicolumn{4}{|l|}{ Ethnicity* } \\
\hline Fon & $4(9)$ & $2(5)$ & $5(12)$ \\
\hline Goun & $15(35)$ & $19(42)$ & $18(43)$ \\
\hline Ouemenou & $1(2)$ & $1(3)$ & $2(5)$ \\
\hline Setto & $2(5)$ & $5(11)$ & $2(5)$ \\
\hline Torri & $7(15)$ & $5(11)$ & $3(7)$ \\
\hline Yorouba & $9(20)$ & 9 (19) & $8(19)$ \\
\hline Other & $6(14)$ & $4(9)$ & $4(9)$ \\
\hline \multicolumn{4}{|l|}{ Education* } \\
\hline No education & $18(40)$ & $18(41)$ & $20(48)$ \\
\hline $1-6$ th grade & $12(28)$ & $14(32)$ & $10(24)$ \\
\hline 7-9th grade & $10(22)$ & 9 (19) & $12(28)$ \\
\hline$>9$ th grade & $4(10)$ & $4(8)$ & 0 \\
\hline \multicolumn{4}{|c|}{ Monthly family income $(\mathrm{XOF})^{\star} \ddagger$} \\
\hline$<15000$ & $1(2)$ & 0 & $1(2)$ \\
\hline $15000-30000$ & $11(25)$ & $11(24)$ & $5(13)$ \\
\hline $30000-50000$ & $8(18)$ & $9(21)$ & $9(21)$ \\
\hline $50000-100000$ & $14(32)$ & $14(31)$ & $17(41)$ \\
\hline$>100000$ & $10(23)$ & $11(24)$ & $10(23)$ \\
\hline \multicolumn{4}{|l|}{ Housing ${ }^{*}$} \\
\hline $\begin{array}{l}\text { Water source/ } \\
\text { compound }\end{array}$ & $21(48)$ & $24(53)$ & $25(59)$ \\
\hline Latrine in house & $43(98)$ & $41(91)$ & $35(84)$ \\
\hline Electricity in house & $35(80)$ & $35(78)$ & $31(74)$ \\
\hline Cement floor & $38(87)$ & $35(78)$ & $36(86)$ \\
\hline
\end{tabular}

${ }^{*} n(\%)$.

+ Mean \pm standard deviation $[n]$.

$\ddagger$ XOF $=$ Communaute Financiere Africaine franc; \$US $1=542.095$ XOF (http://wwp.greenwichmeantime.com/time-zone/africa/benin/currency.htm; accessed 6 January 2006).

malaria. Thus, it is unclear that the observed change in haematological indices in young children in these studies was solely due to the leached iron from the cooking pots.

An in vitro study suggested that iron pots leach iron into cooked food (A Seal, personal communication, 2004). The study examined the iron content of cooked meals in various pots including cast iron, blue steel, stainless steel and aluminium pots. Three types of foods were cooked in the pots - porridge, ugali (maize meal cooked into thick porridge) and beans. The mean iron $\mathrm{mg} / 100 \mathrm{~g}$ of dried cooked food in cast iron pots was similar to blue steel pots. However, the mean iron was lower in ugali compared with porridge and beans. Thus, one explanation for why we did not observe an effect on $\mathrm{Hb}$ or SF concentrations could be because of the consumption of maize-based foods. In our study population $\mathrm{Hb}$ concentrations at baseline were near normal - thus there was almost no room for further iron incorporation in red blood cells. In such cases, we would expect that the iron leached from pots (if bioavailable) would be stored $^{9}$ and result in higher SF concentrations at the end of study (because SF is an index of storage iron) ${ }^{10}$. We did not observe this phenomenon; SF concentrations were significantly lower in the pot groups than in the control group. This questions the bioavailability of the leached iron from the pots, and also questions the generalisability of findings from previous studies to large-scale programmes targeted for the control of IDA through the promotion of iron pots - our results clearly indicate that findings from previous studies are not generalisable to adolescent girls and women of reproductive age.

The cost of iron pots for large-scale distribution was estimated to be US\$3 per pot. In settings where households have two to five people at risk of IDA, the cost per beneficiary would range from US\$0.6 to US\$1.5. This is comparable to Sprinkles ${ }^{\mathrm{TM}}$ and iron tablets. Cost per beneficiary for Sprinkles ${ }^{\mathrm{TM}}$ (60 sachets) is US $\$ 1.2^{11}$, and for iron-folic acid tablets (270 tablets) is US\$0.46 (United Nations Children's Fund programme) and US $\$ 0.27$ (World Health Organization programme) ${ }^{12}$. However, decision-makers who are interested in promoting the use of iron pots must note that iron pots could only be cost-effective when three conditions are met: (1) leached iron from the pots is bioavailable; (2) each household contains two to five people at risk of IDA; and (3) pots are consistently used. That said, there is no evidence at the present time to support the suggestion that iron pots are cost-effective in controlling IDA.

Our results should be used in the context of the study limitations. SF concentrations are falsely elevated in the presence of infection ${ }^{13}$. Another marker for IDA such as soluble transferrin receptor would have been useful. However, we used CRP values to adjust for falsely elevated SF concentrations. Thus, our group comparisons should not be affected by the presence of coexistent infection. Compliance with the pots was measured by self-report and there was no mechanism to adjudicate these results. Thus, we might have overestimated compliance. If true, this implies that the positive re-enforcement by fieldworkers at each monitoring visit was not an effective means for improving compliance - this may be a major factor in achieving a therapeutic benefit. Finally, we did not collect information on nutritional status and dietary intake. However, this should not limit the generalisability of our results to the general population in developing countries.

Iron is an essential nutrient and it is important to consume a balanced diet to maintain sufficient body iron. However, iron deficiency is common among young children, adolescents and women of reproductive age whose iron needs are high ${ }^{14,15}$. Food fortification and supplementation have been used in these subgroups with limited success: the former strategy may not specifically reach these subgroups, and the latter requires behaviour change for adherence to a dosing schedule. Furthermore, other factors that cause anaemia, such as folic acid deficiency and malaria, often coexist in developing countries. 
Table 2 Haematological indices

\begin{tabular}{|c|c|c|c|c|c|c|c|}
\hline & \multirow[b]{2}{*}{ Cast iron pot (IP) } & \multirow[b]{2}{*}{ Blue steel pot (SP) } & \multirow[b]{2}{*}{ Control (C) } & \multirow[b]{2}{*}{ Overall $P^{\star}$} & \multicolumn{3}{|c|}{$P+$} \\
\hline & & & & & IP vs. C & SP vs. C & IP vs. SP \\
\hline \multicolumn{8}{|l|}{$\mathrm{Hb}\left(\mathrm{gl}^{-1}\right) \ddagger$} \\
\hline Baseline & $117 \pm 13$ & $117 \pm 14$ & $115 \pm 15$ & & & & \\
\hline End & $120 \pm 12$ & $120 \pm 14$ & $120 \pm 12$ & 0.5 & 0.73 & 0.47 & 0.91 \\
\hline \multicolumn{8}{|l|}{$\operatorname{SF}\left(\mu \mathrm{gl}^{-1}\right) \S$} \\
\hline Baseline & $40(4.5-148)$ & $40(1.6-213)$ & $42(3.8-215)$ & & & & \\
\hline End & $37(2.6-296)$ & $44(6.3-241)$ & $59(3.4-440)$ & $<0.0001$ & $<0.0001$ & $<0.0001$ & 0.99 \\
\hline \multicolumn{8}{|l|}{ Anaemia } \\
\hline Baseline & $47(47)$ & $51(50)$ & $46(50)$ & & & & \\
\hline End & $45(45)$ & $41(40)$ & $36(39)$ & 0.48 & 0.53 & 0.99 & 0.57 \\
\hline \multicolumn{8}{|l|}{ IDA } \\
\hline Baseline & $10(10)$ & $14(14)$ & $13(14)$ & & & & \\
\hline End & $9(9)$ & $10(10)$ & $3(3)$ & 0.14 & 0.08 & 0.05 & 0.83 \\
\hline \multicolumn{8}{|c|}{$\mathrm{CRP}>8.3 \mathrm{mgl}^{-1}$} \\
\hline Baseline & $15(15)$ & $16(16)$ & $13(14)$ & & & & \\
\hline End & $18(18)$ & $23(22)$ & $9(10)$ & & & & \\
\hline
\end{tabular}

$\mathrm{Hb}$ - haemoglobin; SF - serum ferritin; IDA - iron-deficiency anaemia; CRP - C-reactive protein.

${ }^{*} P$ for overall difference between groups. Models had end values as dependent variable, group allocation and baseline values as independent variables, and household identifier values as random effects. PROC MIXED was used for continuous dependent variables and PROC GLMMIX for dichotomous dependent variables.

$+P$ for pairwise differences between groups; Tukey's adjustment used for multiple comparisons.

$\ddagger$ Mean \pm standard deviation.

$\S$ Median (minimum-maximum).

$n(\%)$

(a)

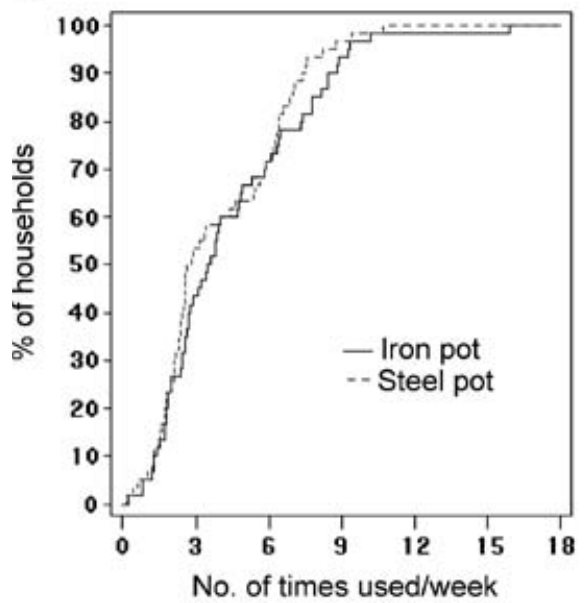

(b)

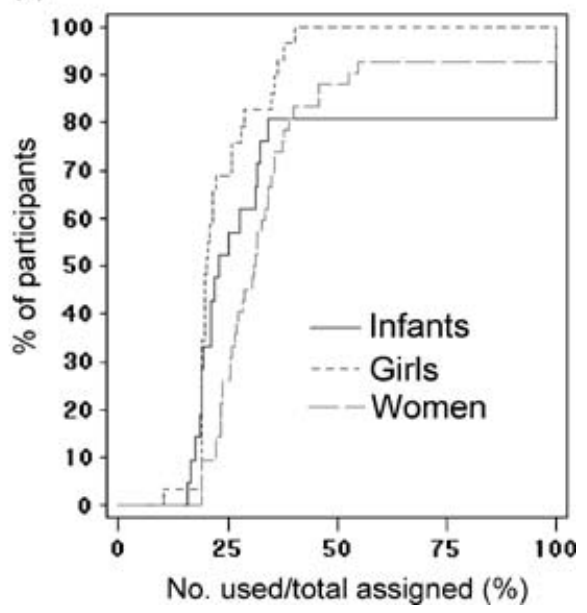

Fig. 2 Cumulative histograms of compliance: $60 \%$ of households used either of the two pots $\geq 3$ times per week (a); $50 \%$ of participants consumed $\geq 20-30 \%$ of the total assigned sachets/tablets, i.e. a total of 60 sachets or 60 tablets (b)

This is believed to be one of the reasons for the limited success of food fortification and iron supplementation in reducing the prevalence of anaemia ${ }^{16}$.

Nevertheless, there is inconclusive evidence that iron pots are effective in controlling anaemia secondary to iron deficiency. Future research on iron pots should focus on studying bioavailability of the leached iron when different types of food are cooked in the pots, in relation to iron supplements of proven efficacy. This can be done by randomly assigning participants to iron pots or control in a cross-over design. Next, serum iron concentrations are serially measured. Finally, the area under the curve for each series of measurements is calculated and, using each participant as his/her own control, relative bioavailability is estimated. A comparable bioavailability would justify further testing of pots in expensive randomised clinical trials, and a low bioavailability would obviate the need for exploring iron pots as an intervention for IDA.

\section{Acknowledgements}

Sources of funding: The study was financially supported by the Canadian International Development Agency (CIDA), and executed by a consortium formed through partnership between CARE Canada, PATH Canada and 
the University of Toronto. CIDA had no role in study design, data collection, data analysis and data interpretation, or in writing the paper.

Conflict of interest declaration: S.Z. Owns the intellectual property rights to micronutrient Sprinkles ${ }^{\mathrm{TM}}$. There are no other competing interests.

Authorship responsibilities: W.S., J.D. and S.Z. participated in designing the study. J.D. participated in acquisition of data. W.S. analysed the data and wrote the first draft of the paper. All researchers contributed to the preparation of and approved the paper.

Acknowledgements: We also acknowledge Michelle Munro and Marlene Barber of CARE Canada, Sian FitzGerald and Peter Berti of PATH Canada, and field staff of CARE Benin for their help in conducting this study.

\section{References}

1 Adish A, Esrey S, Gyorkos T, Jean-Baptiste J, Rojhani A. Effect of consumption of food cooked in iron pots on iron status and growth of young children: a randomised trial. Lancet 1999; 353: 712-16.

2 Borigato EV, Martinez FE. Iron nutritional status is improved in Brazilian preterm infants fed food cooked in iron pots. Journal of Nutrition 1998; 128: 855-9.

3 Hercberg S, Chauliac M, Galan P, Devanlay M, Zohoun I, Agboton Y, et al. Relationship between anaemia, iron and folacin deficiency, haemoglobinopathies and parasitic infection. Human Nutrition: Clinical Nutrition 1986; 40: 371-9.

4 Hercberg S, Chauliac M, Galan P, Devanlay M, Zohoun I, Agboton $\mathrm{Y}$, et al. Prevalence of iron deficiency and iron deficiency anemia in Benin. Public Health 1988; 102: 73-83.

5 Cohen AR, Seidl-Friedman J. HemoCue system of hemoglobin measurement. Evaluation in anemic and nonanemic children. American Journal of Clinical Pathology 1988; 90: 302-5.

6 Chenillot O, Henny J, Steinmetz J, Herbeth B, Wagner C, Siest G. High sensitivity C-reactive protein: biological variations and reference limits. Journal of Clinical Chemistry 2000; 38: 1003-11.

7 Brown K, Lanata C, Yuen M, Peerson J, Butron B, Lonneral B. Potential magnitude of the misclassification of a population's trace element status due to infection: example from a survey of young Peruvian children. American Journal of Clinical Nutrition 1993; 58: 549-54.

8 Geerligs PP, Brabin B, Mkumbwa A, Broadhead R, Cuevas LE. The effect on haemoglobin of the use of iron cooking pots in rural Malawian households in an area with high malaria prevalence: a randomized trial. Tropical Medicine $\&$ International Health 2003; 8: 310-15.

9 Institute of Medicine. Iron. In: Dietary Reference Intakes for Vitamin A, Vitamin K, Arsenic, Boron, Chromium, Copper, Iodine, Iron, Manganese, Molybdenum, Nickel, Silicon, Vanadium, and Zinc. Washington, DC: National Academy Press, 2000; 290-393.

10 Worwood M. Serum ferritin. In: Jacobs A, Worwood M, eds. Iron in Biochemistry and Medicine, II. London: Academic Press, 1980; 203-44.

11 Sharieff W, Horton S, Zlotkin S. Economic gains of home fortification program: evaluation of 'Sprinkles' from provider's perspective. Canadian Journal of Public Health 2006; 97: 20-3.

12 Ladipo OA. Nutrition in pregnancy: mineral and vitamin supplements. American Journal of Clinical Nutrition 2000; 72(1 Suppl.): 280S-90S.

13 Lipschitz DA, Cook JD, Finch CA. A clinical evaluation of serum ferritin as an index of iron stores. New England Journal of Medicine 1974; 290: 1213-16.

14 World Health Organization (WHO)/United Nations Children's Fund (UNICEF). Iron Deficiency Anaemia: Assessment, Prevention and Control. A Guide for Programme Managers. Geneva: WHO/UNICEF, 2001.

15 Zelee Hill Z, Kirkwood B, Edmond K. Family and Community Practices that Promote Child Survival, Growth and Development: A Review of the Evidence. Geneva: World Health Organization, 2004.

16 World Health Organization (WHO)/United Nations Children's Fund (UNICEF). Focusing on anaemia. Towards an integrated approach for effective anaemia control. Joint WHO/ UNICEF Statement [online], 2004. Available at http://www. who.int/topics/anaemia/en/who_unicef-anaemiastatement. pdf. Accessed 22 August 2007. 